\title{
Quantification of Phase Compositions and Diffusional Profiles in Simulated Solid- State Welds of Ti-17 via Super-X Energy-Dispersive X-Ray Spectroscopy
}

\author{
Jonathan Orsborn $^{1,2}$, Robert E.A. Williams ${ }^{2}$, Hamish Fraser ${ }^{1}$ \\ 1. Center for the Accelerated Maturation of Materials, Department of Materials Science and Engineering, \\ The Ohio State University, Columbus, OH, U.S.A. \\ 2. Center for Electron Microscopy and Analysis, The Ohio State University, Columbus, OH, U.S.A.
}

Recent advances in welding technology have enabled the aerospace industry to further reduce the weight of aircraft, by reliably welding titanium alloys. Solid-state welding, in which two metal pieces are welded without either one melting, has recently been proven to produce reliable and consistent welds, and is rapidly becoming the preferred method for joining titanium. Rapid heating rates, short hold-times, plastic deformation, thermal gradients, and fast cooling rates all contribute to the non-equilibrium nature of the process. Thus, a very wide variety of phase transformations, both equilibrium and nonequilibrium, may occur, affecting the microstructure of the welded metal. Since the mechanical properties of titanium alloys are particularly dependent upon microstructure, it is extremely important to understand these phase transformations and the microstructures they produce in order to better inform integrated computational materials engineering(ICME) models. Commercial titanium alloys have two equilibrium phases: the low-temperature hexagonal close-packed (hcp) phase, $\alpha$, and the hightemperature body-centered cubic (bcc) phase, $\beta$. Additionally, non-equilibrium phases, such as martensitic $\alpha$ 'and $\alpha$ ", and athermal and isothermal $\omega$ may also develop depending on thermo-mechanical history [1]. The composition of these various phases and the diffusional profiles that develop during thermo-mechanical processing is a critical piece of information for ICME modeling and a metric that may be quantified more accurately using modern silicon drift detectors for improved XEDS characterization.

This study centers on quantifying the bulk compositions of the various phases present, as well as the diffusional profiles that exist between phases of the alloy Ti-17, or Ti-5Al-4Cr-4Mo-2Sn-2Zr, after being subjected to conditions mimicking those of a solid-state weld. To simulate sold state weld conditions, samples were processed, using a Gleeble ${ }^{\mathrm{TM}} 1500$, to heat round bars at $100^{\circ} \mathrm{C} / \mathrm{s}$ to nearly $1000^{\circ} \mathrm{C}$ and hold for a short time (0-10s). The samples were compressed, while at temperature, then cooled at $\sim 30^{\circ} \mathrm{C} / \mathrm{s}$, similar to the conditions of a solid-state. Imaging in the SEM revealed thin, needlelike intragranular precipitates, on the order of 5-25 nm wide, as shown in Figure 1. However, due to the small size of the precipitates, phase identification was not possible in a bulk, SEM sample. In order to determine the phase of the fine needle precipitates, site-specific TEM samples were prepared using a dual-beam FIB. The TEM lamellae were milled using $30 \mathrm{kV} \mathrm{Ga}^{+}$ions for coarse material removal and 5 $\mathrm{kV} \mathrm{Ga}{ }^{+}$ions for final thinning and clean up. The thin foils were then plasma-cleaned and low-energy $\mathrm{Ar}^{+}$ions milled using a Fischione Nanomill ${ }^{\mathrm{TM}}$, using accelerating voltages of $900 \mathrm{~V}$ and $500 \mathrm{~V}$, for 10 min on each side, at each voltage to remove amorphous damage [2]. TEM analysis was performed using a $300 \mathrm{kV}$ FEI Titan ${ }^{\mathrm{TM}}$ equipped with Super-X XEDS ${ }^{\mathrm{TM}}$ and a monochromator. This combination of technological advances has enabled unprecedented XEDS with high spatial resolution. While the physics of XEDS has not changed, the markedly better signal to noise provided more statistical certainty from quantification. The extreme stability of the stage and the high count-rate capability of Super-X $\mathrm{XEDS}^{\mathrm{TM}}$ system help to enable spatially accurate XEDS measurements, on this ultra small size scale. 
Figure 2(a) displays a HAADF-STEM image of the alpha lath in a beta matrix, while Figures 2(b-f) show the complimentary XEDS compositional maps. Figure 3(a) shows the HAADF-STEM image and a schematic line indicating the line scan direction for Figure 3(b). The XEDS line profile shows the Al concentration to be slightly elevated and $\mathrm{Cr}$ and Mo were reduced in the $\alpha$-phase. The data also showed a build-up of $\beta$-stabilizing elements, in the $\beta$-phase, at the $\alpha / \beta$ interface. This would be consistent with the diffusional nature of $\alpha$-phase precipitating from the $\beta$ matrix. K-factors were also determined experimentally from samples of solutionized Ti-17 to refine the XEDS quantification [3]. To validate and confirm XEDS, the alloy composition was also determined independently via wet-chemical analysis. Experimentally determined k-factors were used to quantify the compositional maps and profiles to reduce the error in quantification. Experimental $\mathrm{k}$-factor determination and quantification refinement will be discussed. [4]

References:

[1] G. Lütjering G. and J.C. Williams in "Titanium", (Springer, Berlin).

[2] R.E.A. Williams, Ph.D. Dissertation (2010).

[3] G. Cliff and G. W. Lorimer, Journal of Microscopy 103 (1975), p. 203-207.

[4] Support for this work, from Tom Broderick at GE Aviation, is greatly appreciated.

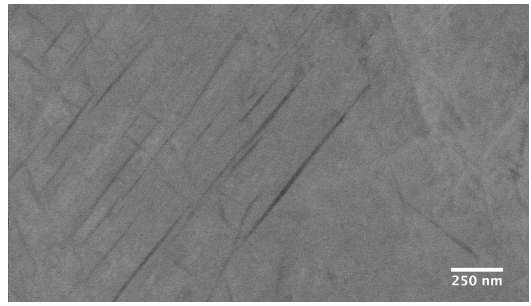

Figure 1. SEM backscattered electron micrograph of the intragranular, needle-like precipitates.
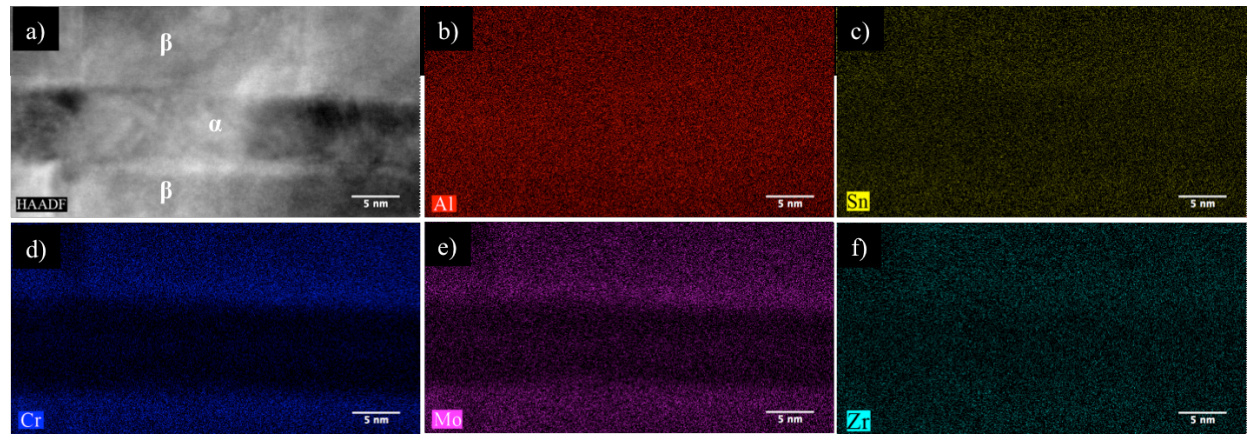

Figure 2. a) HAADF-STEM image of $\alpha$-lath in $\beta$-matrix, with b-f) corresponding quantified elemental maps of solutes (brightness levels were increased, for visibility).
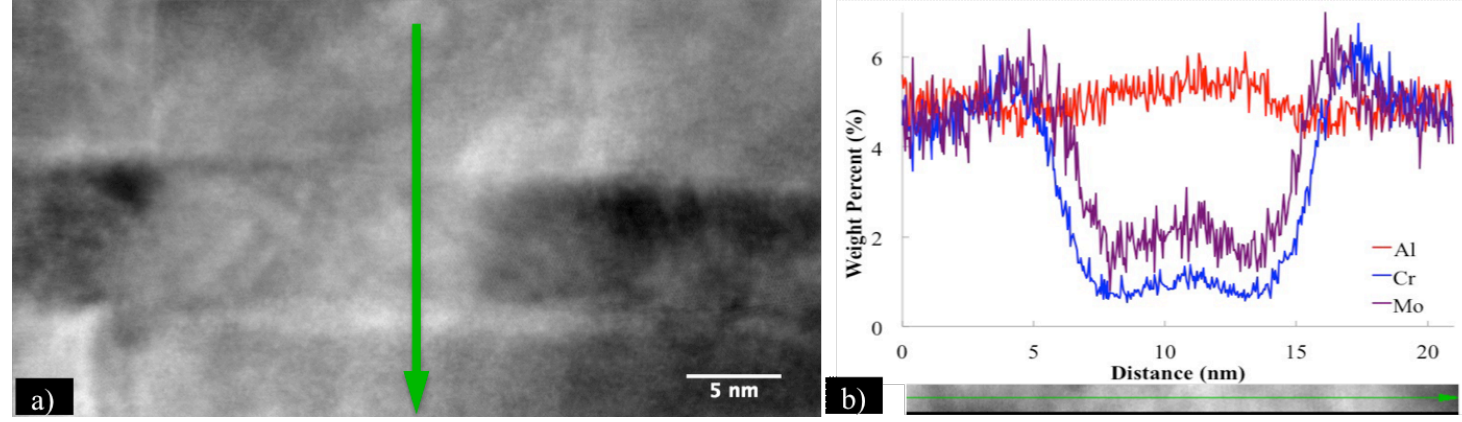

Figure 3. a) HAADF-STEM image showing the line used for b) the compositional profile traversing the $\alpha$-lath (the phase-neutral alloying elements, $\mathrm{Sn}$ and $\mathrm{Zr}$, were not included in the plot for clarity). 